Methods This was a retrospective cohort study of all patients undergoing antireflux surgery from 2001 to 2009 in a tertiary centre under a single surgeon. Patients undergoing fundoplication and/or repair of paraoesophageal hernias were included. Patients underwent preoperative assessment by means of endoscopy, oesophageal manometry and $24 \mathrm{~h}$ oesophageal $\mathrm{pH}$ monitoring. Patients were grouped on the basis of their $24 \mathrm{~h} \mathrm{pH}$ profile into upright refluxers (daytime increased oesophageal acid exposure) and mixed refluxers (night-time increased oesophageal acid exposure +/- daytime reflux). Primary outcomes included if the patient had stopped PPIs following surgery and the incidence of postoperative dysphagia, vomiting and reflux symptoms. Secondary outcomes included if the patients deemed the operation a success.

Results 120 patients were included, with a median age of 49 years (range $24-81)$ at time of surgery. $53 \%(n=63)$ were male and $93 \%$ were laparopscopic procedures. 100 patients (83.3\%) had Nissen fundoplication and $13 \%$ had a combination of fundoplication and paraoesophageal hernia repair. A DeMeester score $>15$ was present in $97.8 \%(n=88)$ patients, with $21 \%(n=19)$ patients having upright reflux and $68.5 \%(\mathrm{n}=63)$ having mixed reflux symptoms on $\mathrm{pH}$ studies. Mixed refluxers were nearly twice as likely to have significant oesophagitis (grade $\mathrm{B}+$ ) and or Barrett's oesophagus on preoperative endoscopy (mixed reflux $57.1 \%$ vs $31 \%$ upright reflux; $\mathrm{p}=0.051$ ). Those patients with mixed reflux symptoms were significantly more likely to stop PPIs postoperatively (mixed reflux $96 \%, n=48$ vs $75 \% n=12$, upright reflux; $p=0.01$ ). Further, the incidence of significant post operative dysphagia was doubled in the upright reflux group (upright reflux $53.3 \%$ vs mixed reflux $26.7 \%$; $\mathrm{p}=0.058)$. Overall, $83.5 \%(\mathrm{n}=76)$ deemed the operation to have been a success, $7.7 \%(n=7)$ a partial success and $8.8 \%(n=8)$ considered that surgery had failed to improve their symptoms.

Conclusion In addition to the DeMeester score for predicting outcomes in antireflux surgery, the presence of reflux only in the upright position may indicate a poorer outcome.

Competing interests None declared.

\section{PTU-191 CIRCUMFERENTIAL RESECTION MARGIN < 1 MM FOLLOWING NEOADJUVANT CHEMOTHERAPY DOES NOT ADVERSELY IMPACT SURVIVAL IN PATIENTS UNDERGOING OESOPHAGECTOMY}

doi:10.1136/gutjnl-2012-302514c.191

${ }^{1} \mathrm{~K}$ Patel, ${ }^{*}{ }^{1} \mathrm{G}$ Bouras, ${ }^{2} \mathrm{R}$ Hejmadi, ${ }^{1} \mathrm{D}$ Alderson, ${ }^{1} 0$ Tucker. ${ }^{1}$ Upper GI Unit, University Hospital Birmingham, Birmingham, UK; ${ }^{2}$ Department of Histopathology, University Hospital Birmingham, Birmingham, UK

Introduction The TNM 7 classification system for oesophageal cancer defines circumferential resection margin (CRM) involvement as R0 ( $>1 \mathrm{~mm}), \mathrm{R} 1 \mathrm{a}(<1 \mathrm{~mm})$, and R1b (involved margin). The prognostic importance of CRM involvement is unclear with previous studies demonstrating conflicting results. We evaluated survival according to CRM status for patients with locally advanced oesophageal adenocarcinoma who had neoadjuvant chemotherapy prior to Ivor Lewis en bloc oesophagectomy with extended lymphadenectomy.

Methods Since 2006, the CRM has been classified as R1a, R1b or R0, assessed by two specialist upper gastrointestinal pathologists in our unit. Since 2007, NAC prior to oesophagectomy was introduced as standard practice in patients with locally advanced oesophageal adenocarcinoma. A retrospective review of prospectively collected data were performed on all patients who underwent oesophagectomy for oesophageal and gastroesophageal junctional adenocarcinoma including demographic data, NAC, final histology, 30, 60, 90 and in-hospital mortality, and survival. Patients with proximal and distal resection margin involvement were excluded. The primary outcome was overall survival evaluated by Kaplan-Meier survival analysis.

Results 63 consecutive resections were included. $62 \%(n=39)$ were R0, 25\% $(n=16)$ were R1a and 13\% $(n=8)$ were R1b. 2-year overall survival was $56 \%, 63 \%$ and $13 \%$ respectively, with a statistically significant reduction in survival between $\mathrm{R} 1 \mathrm{~b}$ and $\mathrm{R} 1 \mathrm{a} / \mathrm{R} 0(\mathrm{p}<0.05)$ Local recurrence rates were similar for R0 (8\%), R1a (19\%) and R1b (13\%). The rate of distant metastases was higher in R1b (75\%) compared to R0 (19\%) and R1a (20.5\%). Univariate analysis demonstrated that poor survival was associated with R1b but not R1a or R0.

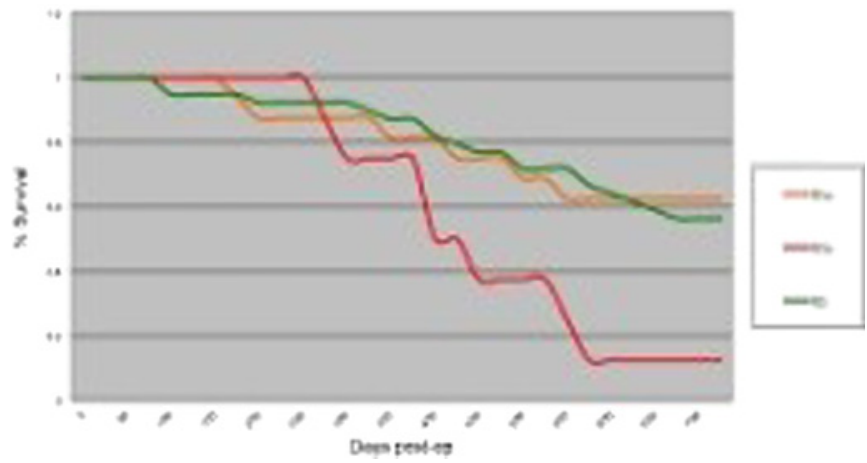

Abstract PTU-191 Figure 1 Kaplan-Meier overall survival according to CRM.

Conclusion R1a and R0 CRM are associated with equivalent recurrence (local and distant) and survival rates to $\mathrm{R} 0$ in patients with oesophageal and GOJ adenocarcinoma following NAC and oesophagectomy. R1a CRM involvement does not adversely survival in patients with oesophageal and GOJ adenocarcinoma following NAC.

Competing interests None declared.

\section{REFERENCES}

1. Pultrum B, et al. A critical appraisal of circumferential resection margins in esophageal carcinoma. Ann Surg Oncol 2010;17:812-20.

2. Sujendran $\mathbf{V}$, et al. Effect of neoadjuvant chemotherapy on circumferential margin positivity and its impact on prognosis in patients with resectable oesophageal cancer Br J Surg 2008:95:191-4.

\section{PTU-192 TIME: PROSPECTIVE STUDY COMBINING ENDOSCOPIC TRIMODAL IMAGING AND MOLECULAR ENDPOINTS TO RISK STRATIFY BARRETT'S OESOPHAGUS}

doi:10.1136/gutjnl-2012-302514c.192

${ }^{1} \mathrm{~K}$ M Shariff, ${ }^{1} \mathrm{M}$ Di Pietro, ${ }^{2} \mathrm{D}$ Boerwinkle, ${ }^{1} \mathrm{X}$ Liu, ${ }^{1} \mathrm{P}$ Lao Sirieix, ${ }^{1} \mathrm{E}$ Walker, ${ }^{1} \mathrm{~L}$ Mills, ${ }^{3} \mathrm{E}$ Telakis, ${ }^{3} \mathrm{~S}$ Slininger, ${ }^{2} \mathrm{M}$ Visser, ${ }^{1} \mathrm{M}$ O'Donovan, ${ }^{3} \mathrm{P}$ Kaye, ${ }^{3} \mathrm{~K}$ Ragunath, ${ }^{2} \mathrm{~J}$ Bergman, ${ }^{1} \mathrm{R}$ C Fitzgerald. ${ }^{1}$ Hutchison-MRC Research Centre, Cambridge, UK; ${ }^{2}$ Academic Medical Centre, Amsterdam, Netherlands; ${ }^{3}$ Digestive Diseases Centre, Nottingham, UK

Introduction Biomarkers have been proposed to improve risk stratification in Barrett's oesophagus (BO), however molecular heterogeneity of $\mathrm{BO}$ can hamper detection of molecular changes in random biopsies. Use of Autofluorescence Imaging (AFI) within endoscopic Trimodal Imaging (ETMI) can improve dysplasia detection, but has high false positive rate. Aims of study were (a) validate biomarkers previously published in separate patient cohorts in single study (b) assess whether AFI can increase detection of biomarkers (c) combine ETMI and biomarkers to improve risk stratification of patients with BO.

Methods Prospective European multicentre study. Each patient underwent ETMI with targeted biopsies on AFI positive (AFI+) areas and one AFI negative (AFI-) area, as well as random 